Atıf için / For Citation: A. Babanl, V. Sabyrov, "1D Kauntum Telin Ortalama Enerjisi ve Öz Isısı”, Süleyman Demirel Üniversitesi Fen Edebiyat Fakültesi Fen Dergisi, 15(1), 7379, 2020.

\title{
1D Kuantum Telin Ortalama Enerjisi ve Öz Isısı
}

\author{
Arif BABANLI ${ }^{1}$, Vepa SABYROV ${ }^{* 2}$ \\ ${ }^{1}$ Süleyman Demirel Üniversitesi, Fen Edebiyat Fakültesi, Fizik Bölümü, 32000, Isparta,Türkiye \\ ${ }^{2}$ Süleyman Demirel Üniversitesi, Fen Bilimleri Enstitüsü, 32000, Isparta, Türkiye \\ *yazlşllan yazar e-posta: sabyroww@gmail.com
}

(Alinış / Received: 19.11.2019, Kabul / Accepted: 03.03.2020, Yaylmlanma / Published: 31.05.2020)

Özet: $\mathrm{Bu}$ çalışmada InAs yariiletken heteroyapıdan oluşan bir boyutlu kuantum telin Rashba spin-orbital etkileşimi ve tel eksenine dik yönde yönelmiş manyetik alan etkisinde elektronların ortalama enerjisi ve öz 1sısı teorik olarak araştırıldı. Sistemin dağılım fonksiyonu hesaplandı. Kuantum tel sistemin ortalama enerjisi, öz 1sısı sicaklığa göre grafiği oluşturuldu ve manyetik alanın şiddetine göre değişimi incelendi. Buna göre kuantum telin elektronlarının ortalama enerjisi, sıcaklık artmakta ve belli bir sıcaklıktan sonra sabit bir değere yaklaşmaktadır. Manyetik alanın şiddetinin artması veya azalması ortalama enerjinin sıcaklığa göre sabit değerini değiştirmektedir. Kuantum teldeki elektronların öz 1sisı düşük sıcaklıkta maksimum değere ulaşmakta ve artan sıcaklıkla birlikte sıfira gitmektedir. Elektronların öz 1sısı sıcaklığa göre manyetik alanın farklı değerlerinde farklı maksimum tepe noktaları vermektedir.

Anahtar kelimeler: Rashba spin-orbital etkileşimi, 1D kuantum tel, Termodinamik nicelikler.

\section{The Mean Energy and Specific Heat of the 1D Quantum Wires}

\begin{abstract}
In this study, Rashba spin-orbital interaction of one dimensional quantum wire consisting of InAs semiconductor heterostructure and the mean energy and specific heat of electrons in the direction of the magnetic field directed perpendicular to the wire axis were investigated. The partition function of the system was calculated. The mean energy of the quantum wire system and its specific heat were plotted according to the temperature and the variation of the magnetic field according to the intensity was examined. Accordingly, the mean energy of the electrons of the quantum wire increases with increasing temperature and approaches a fixed value after a certain temperature. Increasing or decreasing the intensity of the magnetic field changes the constant value of the average energy relative to the temperature. The specific heat of the electrons in the quantum wire shows the maximum peak value at low temperature, then progressively advances towards zero. The specific heat of the electrons gives different maximum peaks at different values of the magnetic field according to the temperature.
\end{abstract}

Key words: Rashba SOI, 1D quantum wire, Thermodynamic quantities. 


\section{Giriș}

Son yıllarda yarıiletken heteroyapıların üzerinde yapılan araştırmalar yeni elektronik cihazların gelişmesine ve üretilmesine neden oldu. Günümüzde hayatımızı kolaylaştıran çok katlamalı heteroyapı güneş bataryaları, tasarruflu olan 1şık yayan LED diyotlar, yüksek frekanslı bipolar transistorlar, alan etkili transistorlar heteroyapılardan oluşmaktadır. Normal p-n tipli yarıiletkenlere göre heteroyapıların genel parametreleri, yani yarıiletkenlerin band aralığı, yük taşıyıcıların etkin kütlesi, elektron enerji spektrumu ve haraketliliği avantajlardan oluşmaktadır. Heteroyapılar ile ilgili ilk teorik araştırma Kroemer tarafindan yapılmıştır [1]. Kroemer kendi çalışmasında p-n homogeçilşere göre heteroyapılarda heterogeçişlerin yüksek enjeksiyon verimliliği olabilir diye hipotezini öne sürdü. Buna ilaveten Kroemer çift katlamalı heteroyapıdan lazer yapılabilir diye fikri ortaya attı [2]. Birkaç yıl sonra AlGaAs-GaAs [3] heteroyapıdan ilk oda sıcaklığında çalışan düşük eşik lazeri (low threshold laser) [4,5], 1şın yayan diyot, heteroyapıdan oluşan güneş foto elementleri, bipolar transistorlar [6] oluşturulmuştur. İlerideki çalışmalar, kuantum kuyularının ve pratik uygulamalarının incelenmesine ek olarak, birçok bilim insanının daha küçük boyutlara sahip kuantum telleri ve kuantum noktalarının çalışmasına ilgi duymasını sağlamıştır. Kuantum tellerde yük taşıyıcı iki yönde sınırlıdır ve sadece tel ekseni boyunca serbestçe hareket edebilen kuantum sistemidir. Tel eksenine göre elektronun enerjisi sabit kalır ama eksene dik yönde enerjisi kesikli değerleri alır. Kuantum tellerin ilk deneysel ve teorik çalışmaları [7] makalesinde araştırılmıştır. [8] çalışmasında teorik olarak kuantum telin optik ve geçirgenlik özellikleri incelenmiştir. Kuantum telli heteroyapılardaki en önemli temel fiziksel olaylardan biri tek boyutlu elektron gazı, keskin tepe noktasina sahip olan durum yoğunluk fonksiyonu ve eksiton bağlanma enerjisinin artış göstermesidir. Katılarda özellikle heteroyapılarda spin-yörünge etkileşimi nedeniyle spin dejenerasyonunun kaldırılmasına getirir. Bu fiziksel olaya Rashba etkisi denir [9-12]. Rashba spin-orbital etkisi spintornik cihazlarının [13] fiziksel temelini oluşturmaktadır. Kuantum telin Rashba spin-orbital ve manyetik alan etkisini optik, spin ve simetrik özelliklerini [14] çalışmasında teorik olarak araştırılmıştır. Kuantum telin termodinamik özellikleri Khordad tarafından incelenmiştir [15]. Khordad kendi çalışmasında dağılım fonksiyonunu $\boldsymbol{k}$ dalga vektörüne bağlı olarak elde etmiştir. $\mathrm{Bu}$ ise termodinamik niceliklerin dalga sayısına bağlı olarak değiştiği anlamına gelmektedir. Bilindiği üzere termodinamik nicelikler dalga sayısından bağımsızdır. Biz kendi çalışmamızda Rashba spin-orbital etkileşimi ve $z$ yönünde yönlenmiş sabit $\boldsymbol{B}$ alana maruz kalmış bir boyutlu kuantum telin dağılım fonksiyonu $\boldsymbol{k}$ dalga vektöründen bağımsız olarak hesaplandı ve buna göre sistemin termodinamik nicelikleri incelendi. Sistemin öz 1s1s1, ortalama enerjisi sıcaklığa göre ve $\boldsymbol{B}$ 'nin farklı değerlerinde grafikleri çizildi.

\section{Materyal ve Metot}

\subsection{Hamiltonyen ve Enerji spektrumu}

1D kuantum tel sisteminin Rashba spin-orbital etkileşimi ve $B$ alan içinde elektronun Hamiltonyeni

$$
H=\frac{1}{2 m}(\boldsymbol{p}+e \boldsymbol{A})^{2}+\frac{1}{2} m \omega_{0}^{2} x^{2}+\frac{\alpha}{\hbar}[\sigma \times(\boldsymbol{p}+e \boldsymbol{A})]_{z}+\frac{1}{2} g \mu_{B} B \sigma_{z}
$$

olarak yazılabilir $[14,16]$. Burada $\boldsymbol{p}$ momentum operatörü, $e$ elektorun yükü, manyetik alanın vektör potansiyeli $\boldsymbol{A}=(0, B x, 0), \sigma$ Paulı matris vektörü, $m$ etkin kütle, $\omega_{0}$ 
sınırlandırıcı potansiyelinin frekansı, $g$ lande faktörü, $\mu_{B}$ Bohr magnetonu, $B$ manytetik alan, $\alpha$ Rashba parametresi. Bu Hamiltonyen için Schrödinger denkleminin çözümü aşağıdaki gibidir [17]:

$$
\begin{aligned}
E_{n}=\frac{\hbar^{2} \Omega}{2 m l_{0}^{2}}\left(\left(2 n+2-\frac{l_{0}^{2} k^{2}}{\Omega}\left(\frac{l_{0}^{4}}{l_{B}^{4} \Omega^{2}}-1\right)\right)\right. \\
\left. \pm \sqrt{\left(\frac{n+1}{2}\right)\left(\zeta_{1} \sqrt{\Omega}+\frac{\zeta_{1}}{\Omega}\right)^{2}+\left(\frac{l_{0}^{2} m g \mu_{B} B}{\hbar^{2} \Omega}-1\right)^{2}}\right)
\end{aligned}
$$

ya-da denklem 2 aşağıdaki gibi yazılabilir,

$$
E_{n}=C_{0}\left((2 n+2-a)+\sqrt{\left(\frac{n+1}{2}\right) b+d}\right)
$$

Burada şu kısıtlamalar yapılmıştır:

$$
\begin{gathered}
b=\left(\frac{l_{0} \sqrt{\Omega}}{l_{S} \Omega}+\frac{l_{0}^{3}}{l_{B}{ }^{2} l_{S} \Omega^{\frac{3}{2}}}\right)^{2}, \quad d=\left(\frac{\left(\frac{l_{0}}{l_{B}}\right)^{2}}{\Omega} \frac{g}{2} \frac{m}{m_{0}}-1\right)^{2} \\
a=\frac{\left(l_{0} k\right)^{2}}{\Omega}\left(\frac{\left(\frac{l_{0}}{l_{B}}\right)^{4}}{\Omega^{2}}-1\right) \\
C_{0}=\frac{\hbar^{2} \Omega}{2 m l_{0}^{2}}, \quad \Omega=\sqrt{1+\frac{l_{0}^{4}}{l_{B}^{4}}}, \quad \omega_{c}=\frac{e B}{m} \\
l_{S}=\frac{\hbar^{2}}{2 m \alpha}, \quad l_{0}=\sqrt{\frac{\hbar}{m \omega_{0}}}, \quad l_{B}=\sqrt{\frac{\hbar}{m \omega_{c}}}
\end{gathered}
$$

denklem 6'deki son üç ifade kuantum telin boyutsuz uzunluklarını karakterize eder [14].

\subsection{Dağılım fonksiyonu}

Kuantum telin dağılım fonksiyonu, denklem 7 ile hesaplanır:

$$
Q=\sum_{n=0}^{N} e^{-\beta E_{n}}
$$


Denklem 7'de $E_{n}$ yerine denklem 3' deki değeri yazılır.

$$
Q=\sum_{n, k} e^{\beta C_{0} a} e^{-\beta C_{0}\left((2 \mathrm{n}+2)+\sqrt{\left(\frac{n+1}{2}\right) b+d}\right)}
$$

Bu ifade-de toplama $k$ ve $n$ kuantum sayılarına bağlıdır. Eğer toplamada $k$ 'ya bağlı terimleri ve $n$ kuantum sayısına bağlı terimleri ayrı toplamalar olarak yazılırsa, denklem 9 ,

$$
Q=\sum_{k} e^{-\beta \frac{\hbar^{2} k^{2}}{2 m \Omega^{2}}} \sum_{n} e^{-\beta C_{0}\left([2 n+2]+\sqrt{\left(\frac{n+1}{2}\right) b+d}\right)}
$$

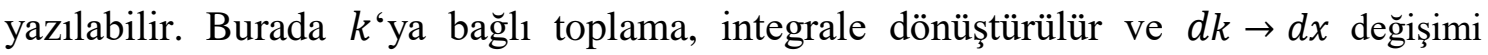
yap1lır $[18,19]$.

$$
\frac{L_{y}}{\pi} \int_{0}^{K} e^{\frac{-\hbar^{2} k^{2}}{2 m \Omega^{2}} \beta} d k \rightarrow \frac{L_{y} m \omega_{0}^{2} \Omega^{2}}{\pi \hbar \omega_{c}} \int_{0}^{\frac{L_{x}}{2}} e^{-\frac{m x^{2} \Omega^{2} \omega_{0}^{4} \times \beta}{2 \omega_{c}^{2}}} d x
$$

Denklem 10'da $x$ 'in değeri,

$$
x=\frac{\omega_{c} \hbar k}{m \omega_{0}^{2} \Omega^{2}}
$$

eşittir [17]. Gerekli matematiksel işlemlerden son denklem 10'un integrali hesaplanarak aşağıdaki değeri elde ederiz,

$$
\frac{L_{y} m \omega_{0}^{2} \Omega^{2}}{\pi \hbar \omega_{c}} \int_{0}^{\frac{L_{x}}{2}} e^{-\frac{m x^{2} \Omega^{2} \omega_{0}^{4} \times \beta}{2 \omega_{c}^{2}}} d x=\frac{L_{y} \sqrt{\Omega^{3}}}{2 \sqrt{\pi} l_{0} \sqrt{\beta C_{0}}} \operatorname{Erf}\left[\frac{\sqrt{\Omega} l_{B}^{2} L_{x}}{2 l_{0}^{3}} \sqrt{\beta C_{0}}\right]
$$

Burada Erf(x) olasilik fonksiyonudur, $L_{y}$ ve $L_{x}$ kuantum telin boyuna ve enine uzunluklarıdır. Denklem 9'da $n$ kuantum sayısına bağlı toplam Euler-Maclaurin toplama formülü kullanarak [20,21].

$$
\sum_{n=0}^{N} f(n)=\int_{0}^{N} f(x) d x-\frac{1}{2}(f(N+1)-f(0))
$$

integrale dönüştürülür ve çözülür. Sonuçta denklem 9'un hesabı, Rashba spin-orbital ve z ekseninde yönlenmiş manyetik alanında olan kuantum telin dağılım fonksiyonu denklem 13 elde ederiz: 


$$
\begin{aligned}
Q=\frac{F}{\sqrt{\xi}} \operatorname{Erf}[P & \sqrt{\xi}]\left(\frac{1}{2}\left(e^{-H \xi}-e^{-\Lambda \xi}\right)\right. \\
& \left.+\frac{1}{8 \xi}\left(4 e^{-I \xi}-4 e^{-\Gamma \xi}+e^{\Delta \xi} \sqrt{b \pi} \sqrt{\xi}(\operatorname{Erf}[Z \sqrt{\xi}]-\operatorname{Erf}[\Theta \sqrt{\xi}])\right)\right)
\end{aligned}
$$

Burada kolaylık açısından boyutsuz parametrelerde şu kısıtlamalar,

$$
\begin{gathered}
F=\frac{\Omega \sqrt{\Omega}}{2 \sqrt{\pi}} L_{y}, \quad P=\frac{\sqrt{\Omega}}{2 y^{2}} L_{x}, \quad \xi=\beta C_{0} \\
H=2+\frac{\sqrt{\mathrm{C} 1}}{\sqrt{2}}, \quad \Lambda=4+2 n+\frac{\sqrt{b+2 d+b(1+n)}}{\sqrt{2}} \\
I=\frac{1}{2}(4+\sqrt{2} \sqrt{\mathrm{C} 1}), \quad Z=\frac{(b+4 \sqrt{2} \sqrt{\mathrm{C} 1})}{4 \sqrt{b}} \\
\Gamma=\frac{1}{2}(8+4 n+\sqrt{2} \sqrt{b+\mathrm{C} 1+b n}), \quad \Delta=-2+\frac{b}{16}+\frac{2 \mathrm{C} 1}{b} \\
\Theta=\frac{(b+4 \sqrt{2} \sqrt{b+\mathrm{C} 1+b n})}{4 \sqrt{b}}
\end{gathered}
$$

yapılmıştır.

\section{Bulgular}

InAs kuantum telin termodinamik niceliklerini hesaplamak için $g=-8$, yük taşıyıcıların etkin kütlesi $m=0.04 m_{0}$, parabolik potansiyelin etkin mesafe uzunluğu $l_{0}=100 \mathrm{~nm}$, rashba sabiti $\alpha=0.375 \mathrm{eVnm}$ ve $N=10$ değerleri kullanılmıştır [15]. Telin boyuna uzunluğu $L_{y}=400 \mathrm{~nm}$ ve enine uzunluğu $L_{x}=4 \mathrm{~nm}$ olarak alınmıştır [17,22]. Kuantum telinin ortalama enerjisi, öz 1sı aşağıdaki formüllerle hesaplanmaktadır.

$$
U=-\frac{\partial}{\partial \xi} \operatorname{Ln} Q \quad C=\frac{\partial}{\partial T} \operatorname{Ln} Q
$$

$\mathrm{Bu}$ formüllere, yukarıda elde ettiğimiz dağılım fonksiyonu denklem 13 yerine koyarak kuantum telin ortalama enerjisi ve öz 1sısı sıcaklığa bağlı grafikleri çizilir.

Şekil 1'de InAs tipi yariiletken kuantum tellerinde elektronların ortalama enerjisinin sıcaklı̆ga göre değişimi gösterilmiştir. Bu grafikte $L_{y}=400 \mathrm{~nm}$ ve enine uzunluğu $L_{x}=$ $4 \mathrm{~nm}$ olan kuantum telin manyetik alan içinde $l_{0}=100 \mathrm{~nm}$ olmak üzere $l_{B}=l_{0}, l_{B}=$ $2 l_{0}, l_{B}=4 l_{0}$ ve Rashba parametresi $\alpha=0.375 \mathrm{eVnm}$ değerlerine bakılmıştır. Grafikten görüldüğü gibi ortalama enerji düşük sıcaklıklarda bir artış göstermektedir ama sıcaklık arttıkça eğriler asimptotik bir değere yaklaşmaktadır. Örneğin, $l_{B}=l_{0}$ eşit 
durumunda sistemin ortalama enerjisi $U=20 \mathrm{~J}$ değerine ulaşmaktadır. Şekil 2'de InAs tipi yariletken kuantum tellerinde elektronların öz 1sısının sıcaklığa göre değişimi gösterilmiştir. Kuantum telin öz 1S1S1 $l_{B}=2 l_{0}, l_{B}=4 l_{0}$ değerlerindeki eğriler hemen hemen aynı tepe noktasına denk gelmektedir. Ama sıcaklık arttıkça $l_{B}=2 l_{0}$ değerindeki eğri $l_{B}=4 l_{0}$ eğrisine göre hızlı bir şekilde sıfıra doğru ilerlemektedir.

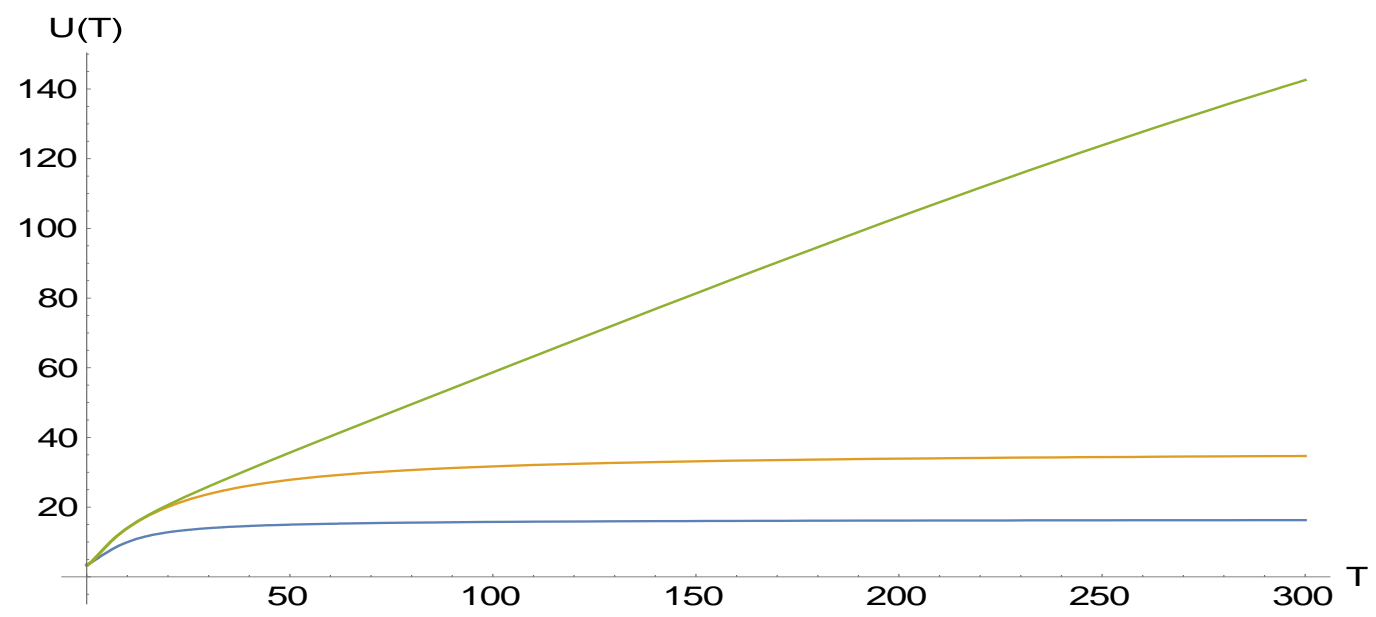

Şekil 1. InAs tipi yarıiletken kuantum tellerinde elektronların ortalama enerjisinin sıcaklığa göre değişimi. Burada $l_{B}=l_{0}, l_{B}=2 l_{0}, l_{B}=4 l_{0}$ sırasıyla mavi, turuncu ve yeşil renkli eğrilerini göstermektedir.

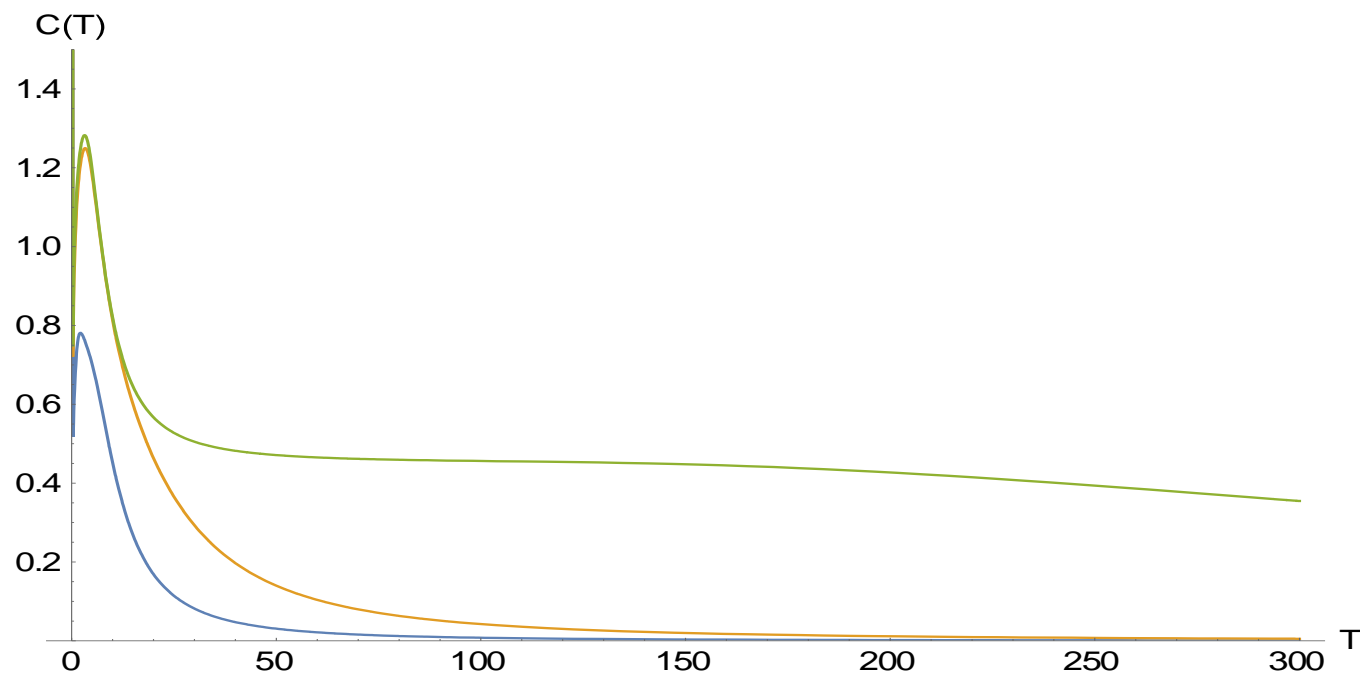

Şekil 2. InAs tipi yarıiletken kuantum tellerinde elektronların öz 1sısının sıcaklığa göre değişimi. $\left(l_{B}=l_{0}, l_{B}=2 l_{0}, l_{B}=4 l_{0}\right.$ sirasıyla mavi, turuncu ve yeşil renkli eğrilerini göstermektedir).

\section{Sonuç ve Yorum}

$\mathrm{Bu}$ çalışmada InAs tipi yarıiletken kuantum telin Rashba spin-orbital etkileşimi ve manyetik alanda elektronların dağılım fonksiyonu hesaplandı ve bundan yararlanarak termodinamik niceliklerin sicaklığa göre değişimi incelendi. Kuantum telin ortalama enerjisi sıcaklık arttıkça artmakta ve sabit bir değere yaklaşmaktadır. Sistemin öz 1sısı sıcaklık arttıkça maksimum değere ulaşıp sonra azalmaktadır. 


\section{Araştırmacıların Katkı Oranı Beyanı}

Vepa SABYROV: Araştırma, Orijinal Taslak Yazımı.

Arif BABANLI: Araştırma, Doğrulama, İnceleme ve Düzenleme.

\section{Destek ve Teşekkür Beyanı}

Bu çalışmanın yazarları olarak herhangi bir destek ve teşekkür beyanımız bulunmadığını bildiririz.

\section{Çatışma Beyanı}

$\mathrm{Bu}$ çalışmanın yazarları olarak herhangi bir çatışma beyanımız bulunmadığını bildiririz.

\section{Etik Kurul Onayı ve/veya Aydınlatılmış Onam Bilgileri}

$\mathrm{Bu}$ çalışmanın yazarları olarak herhangi bir etik kurul onayı ve/veya aydınlatılmış onam bilgileri beyanımız bulunmadı ̆̆ını bildiririz.

\section{Kaynakça}

[1] H. Kroemer, "Quasi-electric and quasi-magnetic fields in non-uniform semiconductors," $R C A$ Review, 18, 332-342, 1957.

[2] H. Kroemer, "A proposed class of heterjunction injection lasers," Proc. IEEE, 51, 1782-1783, 1963.

[3] H. S. Rupprecht, I. M. Woodall, G. D. Pettit," Efficient visible electrolumınescence at $300^{\circ} \mathrm{k}$ from $\mathrm{ga}_{1-x} \mathrm{al}_{x}$ as $p-n$ junctions grown by liquid-phase epitaxy," Appl. Phys. Lett., 11, 81, 1967.

[4] J.I. Alferov, B.M. Andreew, M.K. Turkan, E.L. Portnoy, "Injection lasers based on heterojunctions in an AlAs-GaAs system with a low lasing threshold at room temperature, " FTP , 3, 1328, 1969.

[5] I. Hayashi, M. B. Panish, P. W. Foy, and S. Sumski. "Junction lasers which operate continuously at room temperature,"Appl. Phys. Lett., 17, 109, 1970.

[6] H. Kroemer, "Theory of a wide-gap emmiter for transistors," Proc. IRE, 45, 1535-1537, 1957.

[7] P. M. Petroff, A. C. Gossard, R. A. Logan, and W. Wiegman, "Toward quantum well wires: Fabrication and optical propoties," Appl. Phys. Lett., 41, 635, 1982.

[8] S. Simhony, E. Kapon, T. Colas, D. M. Hwang, and N. G. Stoffel, "Vertically stacked multiplequantum-wire semiconductor diode lasers," Appl. Phys. Lett., 59, 2225,1991.

[9] E. I. Rashba and Al. L. Efros, "Orbital mechanisms of electron-spin manipulation an electric field," Phys. Rev. Lett., 91, 126405, 2003.

[10] E. I. Rashba, "Properties of semiconductors with an extremum loop," Sov. Phys. Solid. State, 2, $1109-1122,1960$.

[11] Y. A. Bychkov and E. I. Rashba, "Properties of a 2D electron gas with lifted spectral degeneracy," JETP Lett., 39, 78, 1984.

[12] R. Winkler, Spin-Orbit Coupling Effect is Two-Dimensional Electron and Hole Systems. Berlin: Springer, 2003, ch 6.

[13] D. Bercioux and P. Lucignano, "Quantum transport in Rashba spin-orbit materials: a review," Rep. Prog. Phys., 78, 106001, 2015.

[14] S. Debald, B. Kramer, " Rashba effect and magnetic field in semiconductor quantum wires," Phys. Rev., B71, 115322, 2005.

[15] R. Khordad, H. R. Rastegar Sadehi, "Low temperature behavior of termodynamic properties of 1D quantum wire under the Rashba spin-orbit interaction and magnetic field," Solid State Communication, 269, 118-124, 2018.

[16] X. W. Zhang, J. B. Xia, “ Rashba spin-orbit coupling in InSb nanowires under transverse electric field,“ Phys. Rev., B 74, 075304, 2006.

[17] A. Gharati, R. Khordad, "Effects of magnetic field and spin-orbit interaction on energy levels in 1D quantum wire: analytical solution," Optic. Quant. Electron, 44, 425, 2012.

[18] F. M. Gashimzade, A. M. Babayev, H. A. Gasanov, " Thermopower of a semiconductor film with parabolic potential in a strong magnetic field," Solid State Physics, 43, 10, 1850-1852, 2001.

[19] B. M. Askerov, Electron Transport Phenomena in Semiconductors. Moscow: Nauka, 1985, ch. 6.

[20] G. M. Fikhtengolts, Differential and integral calculus. Moscow: Nauka, 1969, 7th ed, ch 12.

[21] P. Graham, D. Knuth, O. Patashnik, Concrete Mathematics. Moscow: Mir, 1998, ch 2.

[22] M. P. Stopa, S. D. Sarma, "Parabolic-quantum-well self consistent electronic structure in a longitudinal magnetic field: Subband depopulation,” Phys. Rev. B 40 (14), 10048(R), 1989. 